\title{
Informed consent in clinical trials using stem cells: Sugges- tions and points of attention from informed consent training workshops in Japan
}

\author{
M Kusunose, ${ }^{1}$ MA, MBE; F Nagamura, ${ }^{2}$ MD, PhD; K Muto, ${ }^{1} \mathrm{PhD}$ \\ ' Department of Public Policy, Institute of Medical Science, University of Tokyo, Japan \\ ${ }^{2}$ Department of Advanced Medicine Promotion, Institute of Medical Science, University of Tokyo, Japan
}

Corresponding author: M Kusunose (mayumi.kusunose@fulbrightmail.org)

\begin{abstract}
Informed consent (IC) is an essential requirement of ethical research involving human participants, and is usually achieved by providing prospective research participants (PRPs) with a document that explains the study and its procedures. However, results of a series of IC workshops held in Tokyo during 2014 indicate that consent forms alone are not enough to achieve full IC in regenerative medicine research, due to the necessity of long-term patient-safety observations to meet the ethical challenges of such research. Adequate training of the people who are responsible for obtaining IC (elucidators) is also necessary to ensure full IC. Elucidators must be able to provide PRPs with sufficient information to ensure adequate comprehension of the study and its potential after-effects; judge PRPs' voluntariness and eligibility; and establish and/or maintain partnerships with PRPs. The workshops used role-playing simulations, to demonstrate how to effectively obtain fuller IC, to members of several Japanese research groups preparing for clinical stem cell trials. Workshop results were correlated with the results of a 2013 workshop on what information patients want when considering participation in induced pluripotent stem cell (iPSC) research. The correlated results showed the need for continuous training and education of elucidators in order to make sure that they acquire and maintain IC competency.
\end{abstract}

SAfr J BL 2015;8(2 Suppl 1):49-54. DOI:7196/SAJBL.8016

The world's first-in-human (FIH) clinical trial using induced pluripotent stem cells (iPSCs) was conducted in Japan in 2014. ${ }^{[1,2]}$ Stem cell clinical trials have raised concerns over a variety of ethical issues including: how well prospective research participants (PRPs) have been informed about the nature of the trials, patients' therapeutic misconceptions about the trials, and the need for long-term safety observations of trial participants. These and other scientific issues associated with iPSCs distinguish research in regenerative medicine from ordinary research on therapeutic developments and have led to questions on how well the typical informed consent (IC) process works for these trials.

Informed consent is an essential requirement of ethical research involving human participants, and contains three key components: information, comprehension, and voluntariness. ${ }^{[3]}$ Usually IC is achieved by providing PRPs with a document that explains the study and its procedures. However, transplanted cells differentiated from iPSCs as they were genetically manipulated and long-term observation of trial participants is mandatory for early detection of unknown side effects. We need to put greater emphasis on informing participants about the nature of the trial and the potential after-effects. Informed consent documents cannot always anticipate all participant informational needs, increasing the importance of question and answer sessions between PRPs and those responsible for obtaining full IC.

One way to address IC incompleteness is establishing standards for IC documents and procedures. Several organisations have suggested standards and guidelines for more fully informing PRPs in order to ensure a clear understanding of IC associated with stem cell research.
The International Society for Stem Cell Research (ISSCR) propose the following guidelines:

- Patients need to be informed when stem cell-derived products have never been tested before in humans and if researchers do not know whether they will work as hoped.

- Cell-based interventions, unlike many pharmacological products or even many implantable medical devices, may not leave the body and may continue to generate adverse effects for the lifetime of the patient. The possible irreversibility of a cellular transplant should be explained clearly.

- Subjects should be informed about the source of the cells so that their values are respected.

- Ensuring subject comprehension must be done at each phase of the clinical trials process. Ideally, the subject's comprehension of information should be assessed through a written test or an oral quiz during the time of obtaining consent.

- Human subjects' research committees should ensure that informed consent documents accurately portray these uncertainties and potential risks, and clearly explain the experimental nature of the clinical study. ${ }^{[4]}$

Aalto-Setälä et al ${ }^{[5]}$ suggest guidelines that permit PRPs' participation in stem cell research only if they agree to specific conditions: genetic modification of cells; injection of iPSCs or their derivations into nonhuman animals, including injections into the brain; sharing cell lines with other researchers with appropriate confidentiality protection; and patenting scientific discoveries and developing commercial tests and therapies, with no sharing of royalties with cell donors. 
Each recommendation brings different ethical concerns to any discussion about IC reforms. The many ethical problems revealed in the recommendations show that improving IC related to stem cell research is a pressing issue that needs to be resolved to protect research participants.

\section{Purpose}

This paper examines the results of a series of workshops conducted in 2014 to determine specific areas of concern regarding IC and to identify methods to address these concerns. Workshop results are then compared to a 2013 workshop that studied what information is wanted by patients when considering participation in iPSC research. Each study was concerned with different aspects of the role elucidators play in achieving full IC. For the purpose of this paper, we have defined 'elucidators' as 'people who obtain and are in charge of IC', even though IC is usually conducted by investigators, physicians, research nurses, or clinical research coordinators.

\section{Method}

Workshops on IC in clinical trials using stem cells were conducted in Tokyo in February and November 2014. We accepted applications for the workshops from Japanese research groups enrolled in the 'Highway Program for the Realization of Regenerative Medicine', funded by the Japan Science and Technology Agency, who planned to conduct stem cell clinical trials in the near future. All participants conduct or support the IC process for PRPs or prepare consent forms for their research groups.

Prof. Sean Philpott-Jones, Director of the Bioethics Program/ Director of the Research Ethics Program for the Bioethics Program at Union Graduate College - Icahn School of Medicine at Mount Sinai in New York, was invited to lead the workshop. Prof. PhilpottJones has extensive experience with IC education using role-play methods. As part of the workshop preparation process, consent forms were carefully drafted to incorporate comprehensive information related to IC (Table 1) and the forms were distributed to the workshop participants (WPs) a week before the workshop began.

The February workshop served as a pilot study to obtain feedback from participants regarding workshop forms and procedures. Seven people participated in the four-and-a-half hour workshop. One male and one female from a group of volunteers, helping medical students prepare for the Objective Structured Clinical Examination (OSCE), acted as patients. WPs acted as IC elucidators. Two consent forms were prepared for the workshop:'Transplant of Chondrocytes Derived from Autologous Bone Marrow Mesenchymal Stem Cells (MSCs) for Cartilage Damage in Knee Joints (Phase I Trial)' (educational material) and 'Clinical Research on Autologous iPS Cell-Derived Retinal Pigment Epithelium Sheet Transplantation for Exudative Age-Related Macular Degeneration' (a real consent form from the clinical trial).

Feedback, following the pilot workshop, led to the creation of a new IC form for the 'Transplant of Chondrocytes' and to the recruitment of better performers as workshop patients.

In November, six people participated in a six-hour workshop. Two of the six had participated in the first workshop. For this workshop two female actors from an entertainment agency played the patients. As part of using the new IC form, bogus patient information and referrals were created to preserve confidentiality.

\section{Table 1. Drafting consent forms}

- Introduction

- What is a clinical trial?

- Cartilage damage in knee

- Treatment of the knee joint

- About this clinical trial and its objective

- Methods of the clinical trial

- Selection criteria for research participants of this clinical trial

- Period and planned number of participants of this clinical trial

- Study site

- Study method

- Risks

- Treatments other than this clinical trial

- Treatments after completion of this clinical trial

- Benefits

- Disadvantages

- Expense for participation in this clinical trial

- Treatment and coverage for health hazards caused by this clinical trial

- Privacy protection

- When we obtain new information on this clinical trial

- Consent for participation in this clinical trial

- Right of consent withdrawal

- Discontinuation of this clinical trial

- Specimen storage for safety check

- Access to records

- Intellectual property right

- Conflict of interest

- Funding sources of this clinical trial

- Contact information and consultation service

- Complaints counter

- Clinical research coordinators

- Address of discontinuation request

Both workshops used role-playing to teach how to obtain IC from PRPs. Volunteers and/or actors played patients. Characteristics, background, and medical conditions of the 'patients' were created in detail beforehand so that the translational-research physician could play their parts realistically. In the workshop held in November (the MSC case), one actor played a woman whose husband had told her to participate in the trial so that she could get better and do more housework (Fig. 1). In the iPSC case, an actor played a young actor who did not want surgical scars on her body because of her occupation and was also struggling financially, which made her eager to try clinical trials; the character also wished to have a baby in the near future (Fig. 2). Both actors were required to ask specific questions, such as could they participate if pregnant, and were encouraged to ask for explanations for any jargon used during the session. They were also free to ask questions about anything else that they believed would help them decide whether to participate (except for specific medical issues that the clinical trials were not designed to treat). 


\section{$11 / 07 / 2014$}

Dear Professor Taro Bando,

I would like to discuss one of our patients, Ms. xxxx. Though she has been treated at our hospital as an outpatient due to cartilage damage in her knee joints, she has also received conservative medical therapy as described below. However, she is now in thinking of surgical therapy due to disabling symptoms and other occupational reasons. Alternative therapies are autologous transplantation ('mosaicplasty'), in which a part of the non-damaged knee cartilage is obtained and transplanted into the damaged area in order to facilitate the regeneration of hyaline cartilage; the use of autologous cultured cartilage called JACC which has been approved as regenerative medicine; and artificial joint replacement. I have also suggested transplantation of chondrocytes derived from autologous bone marrow mesenchymal stem cell for cartilage damage in knee joint. When I told her about the research that was done with the second research subject, she told me she would like to hear about your clinical trial. I gave her a brief explanation but did not hand her a copy of the consent form. I would appreciate it if you could inform her about the clinical trial.

\section{Patient information}

Name: xxxxxxxx, Age 46 y/o, ID \# xxxxxxxxx

Contact information: Address: xxxxxx Phone: \# xxxxxx

Occupation: Tea ceremony instructor (runs a tea ceremony school at home)

Family history: Husband (physician), son, daughter, no special instructions

Anamnesis: No special instructions

\section{Progress}

Six months ago, when walking, she was knocked down and injured by a motorcycle driver. Bruise and torsion caused by the fall led to three days of hospitalization, during which the affected area was immobilized and kept under observation. Currently, she is under treatment for tibial cartilage damage as an outpatient.

For cartilage damage, the following are conducted: internal use of anti-inflammatory analgesic, intra-articular administration of steroid and hyaluronan once a week, and drainage of joint fluid when needed. Tumentia is slight; however, she complains of severe pains whenever she has to move about.

\section{Special instructions}

She is a tea ceremony instructor and is eager to continue managing her tea ceremony school. However, she cannot go down on her knees due to the limitations in her knee range motion, and so she is face with the prospect of closing her business. Therefore, she wants therapy so that she can continue to work as an instructor at her tea ceremony school. Her husband is a physician. It seems that he is positively reinforcing her participation in the clinical trial and expecting her early recovery. She, on the other hand, has a propensity for anxiety, and she appears to have a fear of invasive therapy such as removal of autologous cartilage.

\section{Professor Kazuo Ohmori, MD Orthopaedist}

Fig. 1. Patient information sheet - patient 1

\section{$11 / 07 / 2014$}

Dear Professor Bando,

I would like to introduce a patient, Ms. xxxx. She is in outpatient treatment at our hospital for cartilage damage in the keen joint, sustained in a traffic accident. Thus far, she has received conservative medical therapy; however, due to her disabling symptoms, I suggested to her that at this stage she might consider seeking surgical therapy. I explained a number of potential therapies, as follows: 1) anaplasty of the damaged area using an arthroscope (reducing physical stimuli by removing processus), 2) drilling or microfracture (methods of bone marrow stimulation, which arthroscopically resect the subchondral bone), 3) mosaicplasty (autologous transplantation of knee cartilage), 4) autologous cultured cartilage called JACC which is a medical product for regenerative medicine and 5) artificial joint replacement. My recommendation was that the final option, artificial joint replacement, is the most realistic; options 1-4 would not offer a comprehensive remedy, due to the large size of the damaged area. However, Ms. xxxx is still young. If she opts for artificial joint replacement, she will require several additional replacement surgeries in the future, which will result in large scars. Additionally, bone malformation caused by the artificial joint will cause surgical difficulties to increase with each operation. Ms. $x x x x$ is an active professional actor, and as such wants to keep scarring to a minimum. She is interested in treatment via chondrocytes derived from autologous iPS cells, a procedure she heard of through the press. She has stated that she would like to learn more about your clinical trial. I have explained the trial broadly, but have not given her a copy of the consent form. Treatment options 3 and 4 will be difficult to conduct because of the extent of the damaged area; however, I assume that it is possible to secure the necessary cell numbers if regenerative therapy with using iPS cells. I have also explained to her that there is no one who received this method. I thank you in advance for your support.

\section{Patient information}

Name: xxxxxx Age: 29 y/o ID \#: xxxxxx

Contact address: $x x x x x x$ Contact phone \#: xxxxxx

Occupation: Actor

Family history: Single / no special instructions

Anamnesis: No special instructions

\section{Progress}

Three months ago, the patient collided with a vehicle while bicycling. She was hospitalised at our facility to immobilise the affected area, and received follow-up care for two weeks. Currently, she is under treatment as an outpatient.

In the outpatient department, the following therapies are conducted: administration of internal anti-inflammatory analgesics; once weekly trial implementation of intra-articular steroid and hyaluronan administration; and drainage of joint fluid as needed. Tumentia is slight, but she complains of severe pain, particularly in movement, which interferes with her daily life.

\section{Special instructions}

Regarding her therapy, she does not want to be left with visible scars on account of her occupation. Currently she has no choice but to suspend her work as an actor, which causes her financial concern; she wants to return to her business as soon as possible. Although thus far she has focused on her profession, she has a boyfriend and is now considering future pregnancy. When I discussed your clinical trial with her, she had a positive outlook.

\section{Professor Kazuo Ohmori, MD} Orthopaedist

Fig. 2. Patient information sheet - patient 2 


\section{Table 2. Points of attention for informed consent}

- SITUATION: Informed consent should be conducted not in places which conjure images of medical treatment such as a medical examination room. Thus, white coats should not be worn to avoid prospective participants' misunderstanding the process for therapy.

- PERCEPTION: It is important to understand what patients know and expect.

- INFORMATION: Information should be delivered without using scientific jargon and technical terms so that it can be understood by prospective participants.

- KNOWLEDGE: Make sure that prospective participants gain enough knowledge throughout the IC process to assess the meaning of becoming research participants and what would happen to them while participating in research.

- EMPATHY: Try to understand prospective research participants' emotion such as fear and hope. Thus, always pay attention to not only prospective research participants' words but also to their body language and attitudes and tones.

- SOLUTION: Regardless of obtaining prospective research participants' agreement or not, make sure that they understand the next procedure after they leave the room, such as making the next appointment for medical treatment or giving them contact address and advising them to consult with their family members. Also, people who conduct informed consent need to be aware that they are responsible for judging participation eligibility in the research of patients. ${ }^{\left[{ }^{[6]}\right.}$

The workshops began with a lecture detailing six points important for IC: situation, perception, information, knowledge, empathy, and solutions (Table 2). ${ }^{[6]}$ Then mock research protocols were introduced, followed by patients information, and a questionand-answer session. The WPs then explained the research to each mock patient as part of comprehensive IC. The lectures and WPs' performances were videotaped, and used to review their performances.

Workshop results were then compared to the results obtained in a 2013 iPSC workshop conducted by the Japanese Retinitis Pigmentosa Society (JRPS) that featured a dialogue between retinitis pigmentosa patients and researchers. Correlating workshop results enabled both WP and PRP perspectives to be incorporated into recommendations for improving IC related to stem cell research.

\section{Results \\ WP performance in the workshops}

Although WPs performed well overall, they had difficulty with four of the six points deemed necessary for full IC: perception, information, knowledge, and empathy.

\section{Observations regarding WP performance}

- WPs tended to take too much time explaining what they believed to be important or familiar, such as medical conditions and risks.

- WPs provided the information requested on the consent form, but did so in a scattered order, based on the patients' questions.

- WPs paused sessions to get answers from the translationalresearch physician for questions they could not anwer, such as medical procedures. The WPs then tried to explain the information to PRPs.

- WPs who lacked IC training in the research context found it particularly difficult to explain research processes without using jargon.

- SomeWPs failed to notice non-verbal signs such as facial expressions or body language as indications of a patient's understanding or concerns. For instance, in the case of the woman pressured by her husband, one WP neglected to suggest to the patient that it should be her decision to participate in the trial.

- Some WPs covered information too fast for the PRPs to follow because they focused too much on 'explaining.'

\section{Suggestions for improving WP performance}

- If an elucidator takes too long to explain important aspects of a study it may imply bias regarding the PRP's ability to participate in the study. For instance, if the elucidator highlights benefits or risk excessively, a PRP might think the elucidator is implying that he or she should or should not participate in the research. Manipulation, coercion, and misrepresentation should be avoided during IC. When explaining the research to PRPs, an elucidator should be careful not to misrepresent the research by overestimating benefits and underestimating risks. Even the order in which items are explained could affect a PRP's decision. The elucidator should provide information in a neutral manner so that PRPs can make decisions based on their own thoughts and values.

- A well-written consent form should be a 'roadmap' ensuring all of the necessary elements are explained during IC. Elucidators should follow the order of the items listed on the consent form. It is during the icebreaking stage that: elucidators can ask PRPs about their reasons for participating in the research; their expectations and concerns; and allow the PRPs to ask questions and express their thoughts and feelings. Elucidators should always organise the meetings in ways that ensure all important information is conveyed.

- WPs tended to think they had to answer every question that the PRPs asked. Some tried to provide medical explanations, which should be left to attending doctors to do. Elucidators should admit their lack of knowledge when asked a question beyond their expertise. The elucidator's duty is not to provide information he or she is unfamiliar with but to advise PRPs to seek medical information or ask medical doctors for options before deciding to take part in a clinical trial. Elucidators should use the PRPs' questions to assess their comprehension, educational level, scientific and medical knowledge, and home background, and assess why PRPs want such information. Elucidators must understand that they cannot always adequately address patients' questions and concerns during the initial recruitment and consent process. They must recognise that IC is a process not a onetime event. They must continually engage PRPs in discussions of the trial design, conduct, and outcomes throughout the research process, especially for studies involving cutting-edge interventions.

- Information should be explained using simple words and easily understood expressions. In our workshop, those involved in stem 
cell research and IC support sometimes found it difficult to explain scientific matters without using technical terms. PRPs often misunderstand that the purpose of a phase-I clinical trial is to evaluate safety, not to find a cure. Elucidators must be able to explain research without causing therapeutic misconceptions and to ensure PRPs' comprehension in a limited amount of time.

- Elucidators must build their empathy skills and be able to create a rapport that enables PRPs to voice their concerns and ask questions, and enables elucidators to recognise the non-verbal communication signs expressed by PRPs about the information being communicated or other factors that may influence their decision-making.

\section{What information do research volunteers want to know?}

In 2013, the Japanese Retinitis Pigmentosa Society (JRPS) held a workshop for regional leaders. After hearing lectures on iPS research, the research process, ethical issues (including therapeutic misconceptions), and participants' rights and duties, the patients and their families were divided into eight groups of six members to discuss what information they would like when considering participating in a clinical trial. Following is a summary of the information that patients wanted, identified from their comments in the workshop report. ${ }^{[7]}$

- Foreseeable risks and problems, including information on adverse events:

'[I] would like to know [if it is] possible [for this cancer to] spread all over the body, [and if so, should]...the whole or part [of] the eye...be [removed] ? ${ }^{\text {[7] }}$

- Expected burdens and restrictions in daily life, including economic and family burdens:

.... I would like to know if there is something that [will] restrict my daily life during the follow-up period.... First of all, we would like to get better and contribute to the research. However, I am wondering if I have to] abstain from alcohol entirely for three years thereafter. ${ }^{[7]}$

- Physical, psychological, and economic support, including followup care and insurance when an adverse event occurs:

'We would like to know what kind of care would be provided for each adverse event. We are informed of possible risks in advance, but we would like to know what concrete care [is offered for] each specific adverse event....We would like to know... the care [offered] if someone's eyesight was impaired or [he or she] became blind.... We would [also] like to know the medical treatment and/or economic support [offered] for physical symptoms or mental aberrations if they happen. ${ }^{\text {[7] }}$

- Other studies or alternative therapies: One PRP's concern was whether participating in certain research would limit access to other studies or therapies, or whether participants could easily switch to alternative options if they changed their mind.

... we would like to know if other... research [is currently being conducted], not limited to iPS cells, but [interventions] such as artificial retinas and gene therapy. We would like to know of...[issues such as] expected future effects. ${ }^{\text {17] }}$
- Previous PRP results and experiences: From a scientific perspective, it is preferable not to share results from previous research participants. However, based on the dialogue and our experiences, patients want such information when they are making their decisions.

'Second is the information about [previous PRPs' experiences] in the clinical trial. We would like to know if and when ... previous research participants' results [will be available] ... ${ }^{\text {i] }}$

- What patients can do to prevent adverse events so that they are not disqualified as research participants.

$\therefore$... we would like to [know] what we should pay attention to in daily life so as not to cause adverse events [that could] prevent us from [continuing to] participate in the research. ${ }^{[1]]}$

Patients also wanted other information, such as:

- Why they were selected as research participants

- Expected realistic benefits

- Schedules, test items, and restrictions

- Treatments and support, if they developed research-unrelated diseases

- Restrictions related to pregnancy.

Finally, the patients had requests and suggestions on the research on retinitis pigmentosa:

- Suggestions for research designs from a scientific perspective

- Requests for consultation services provided by a third party

- Requests for thorough cell-quality management, such as contamination prevention

- Requests for a mutual-check system of institutional review boards involving representatives from patient groups

- The necessity for educational activities conducted by and for the patients themselves.

Overall, the patients were concerned about how participating would impact their daily routines and quality of life, and wanted concrete, precise information before making a decision. Well-written consent forms usually include much of the information desired by patients, although patients often want more informational certainty than can be provided.

\section{Discussion}

Many studies have been conducted on improving consent forms and readability as well as assessing PRPs' comprehension, such as the ISSCR guidelines, which propose a written test or an oral quiz during $\left.\right|^{\mid C}{ }^{[4]}$ In those cases, the focus is on PRPs' comprehension and scientific literacy. However, stem cell research is very technical and, as with much medical research, there is information asymmetry between laypeople and elucidators, particularly where those elucidators are researchers. Hence, relying solely on written consent forms or educating the PRP in initial IC is not enough to achieve IC. Results from the IC workshops discussed in this paper suggest that improving elucidators' IC competencies is another important and essential element in achieving IC.

The competencies required for competent elucidators include not only an ability to explain the research and enhance PRPs' understanding, but also an ability to judge PRPs' voluntariness and 
eligibility to participate in the research. Out of thousands of patients, only a few people will be selected to enrol in an iPSC-FIH clinical trial and to establish iPSC lines, at a cost of tens of thousands of yen per patient. ${ }^{[7]}$ Those selected PRPs carry the hopes of patient groups, researchers, and the country, putting them under implicit and explicit pressures, especially if they want to withdraw from the trial. ${ }^{[7]}$ Thus, securing research participants' voluntariness and ensuring the right to withdraw from the research is a significant role for elucidators.

Sharing research results with participants is also a challenging issue for elucidators. Various medical examinations are conducted in the process of stem cell research, such as screenings for infectious diseases and cancers, whole genome sequencing, among others. If minors are included as PRPs, a positive result for a sexually transmitted disease may impact the protection of his or her confidentiality as an adolescent, along with parental responsibility. Cancer screenings would also be conducted prior to a PRP being cleared to participate, ${ }^{[7]}$ and whole genome sequencing might be conducted to maintain the quality of cell products for allogenic cell therapy. Obviously, these test results could affect a PRP's quality of life. In order to competently deal with such diverse issues, elucidators must be able to catch signs of anxiety and concern in PRPs during $\mathrm{IC}$, as expressed verbally and non-verbally by the participants. This requires astute communication skills, not often learned by researchers.

Elucidators also need an ability to establish and maintain partnerships with both PRPs and investigators to ensure long-term safety. Monitoring and ensuring the long-term safety of both active research participants and those who withdraw from the study is also important, due to the essential nature of stem cell research. It may not be possible to remove the implanted cells from the body of either active or inactive patients. An elucidators' ability to deal appropriately with people in the multiple contexts of stem cell research is another crucial element in protecting research volunteers.

In summary, elucidators need to act not only as information providers assisting PRPs with decision-making, but also as gatekeepers and coordinators. The complexity of their responsibilities will grow as stem cell research advances. Continuous training and education is essential for elucidators to acquire and maintain such complex skill sets and IC competencies in stem cell research. As demonstrated by the workshops, role-playing can be a practical method of transmitting such knowledge and IC competency.

Due to the limitations of our study, additional research is required to confirm whether there are any traits specific to stem cell research in general. Further research is also necessary to confirm what items should be included in elucidators' IC competency.

\section{Conclusion}

In our IC workshops, we could not find any specific IC requirements for stem cell research. Well-written consent forms cover most of the information that research volunteers want to know. However, relying only on consent forms is not enough to achieve IC. The IC competency of elucidators is another element in protecting research volunteers. The elucidator's role requires skill at explaining the research, enhancing PRPs' understanding, and judging PRPs' voluntariness and eligibility to participate in the research. The need for ongoing education and training of elucidators is essential to their acquiring and maintaining the skill sets needed for them to fulfil their complex role in obtaining full IC for stem cell research.

Conflict of interest. The IC workshops and the dialogue session mentioned in this paper were funded by the Japan Science and Technology Agency (JST). The authors are involved in ethical support for the Research Center Network for the Realization of Regenerative Medicine, which is organised by JST.

Acknowledgments. Foremost, we would like to express our sincere gratitude to Prof. Sean Philpott-Jones, Director of the Bioethics Program/Director of Research Ethics Program for the Bioethics Program at the Union Graduate College - Icahn School of Medicine at Mount Sinai, for giving practical and valuable instruction during our IC workshops. We also extend our gratitude to Prof. Masayo Takahashi at the RIKEN Center for Developmental Biology for letting us use the consent form from her clinical trial in our workshop. We would also like to thank all participants, Prof. Etsuko Arita at the School of Pharmacy, Kitasato University, and the staff who took part in the 2014 IC training sessions. Last but not least, we would like to express gratitude to Prof. Robert Congleton of Rider University for his helpful suggestions.

\section{References}

1. Reardon S, Cyranoski D. Japan stem cell trial stirs envy. Nature, 16 September 2014. http://www.nature.com/news/japan-stem-cell-trial-stirs-envy-1.15935 (accessed 5 August 2015)

2. Kyodo J. Japanese team first to use iPS cells in bid to restore human sight. Japan: The Japan Times, 12 September 2014. http://www.japantimes.co.jp/ news/2014/09/12/national/science-health/japanese-ips-based-retinatransplant-a-global-first/\#.VeEtRfmvGUk (accessed 5 August 2015)

3. The National Commission for the Protection of Human Subjects of Biomedical and Behavioral Research. The Belmont Report: Ethical Principles and Guidelines for the Protection of Human Subjects of Research. United States of America: US Department of Health and Human Services, 1979. http://www. hhs.gov/ohrp/humansubjects/guidance/belmont.html\#xinform (accessed 1 June 2015)

4. International Society for Stem Cell Research. ISSCR guidelines for the clinical translation of stem cells. ISSCR, 2008 http://www.isscr.org/docs/default-source/clin-trans-guidelines/ isscrglclinicaltrans.pdf (accessed 26 February 2015).

5. Aalto-Setälä K, Conklin BR, Lo B. Obtaining consent for future research with induced pluripotent cells: Opportunities and challenges. PLoS Biology 2009; 7(2):e42. [http://dx.doi.org/10.1371/journal.pbio.1000042]

6. Philpott-Jones S. Lecture. In: Kusunose M, Muto K, Philpott-Jones S. Informed consent in clinical trials using stem cells: Suggestions and points of attention from an informed consent training workshop with stimulated patients [Poster] ISSCR 2014 Annual Conference, February 2014

7. Muto K, Takahashi M and Japanese Retinitis Pigmentosa Society (JRPS). Cocreating clinical trials - From a dialogue between patients and researchers [Report in Japanese]. Tokyo: JRPS \& Department of Public Policy at the Institute of Medical Science, University of Tokyo, 2014. 